\title{
Long Life, High Performance Anti-Reflection Treatment for HgCdTe Infrared Focal Plane Arrays
}

\author{
Bruce D. MacLeod ${ }^{*}$ and Douglas S. Hobbs \\ TelAztec LLC, 15 A Street, Burlington, Massachusetts 01803 USA
}

\begin{abstract}
The image contrast and clarity recorded by backside illuminated $\mathrm{HgCdTe}$ focal plane arrays (FPAs) is strongly dependent on minimizing signal loss and detector noise caused by scattered and reflected light from the FPA window and imaging optics. Thin film anti-reflection (AR) treatments based on stacks of thin-film materials have been exclusively used to minimize substrate reflections for this application. The performance and lifetime of these thin-film AR coatings is limited, and can be inadequate for some space based applications due to the damage produced in the coatings by radiation exposure and extreme temperature variations.

A new type of high performance AR treatment for $\mathrm{HgCdTe}$ FPAs promising very wide bandwidth operation and increased lifetime in high radiation environments is under development as part of the MDA's Space Tracking and Surveillance System, or STSS. Based on surface relief microstructures fabricated directly in the FPA window, the new textured AR treatment replaces thin-film coatings, eliminating inherent coating limitations such as stress, thermal expansion mismatch, adhesion, radiation hardness, and low laser damage thresholds. Progress on the design, fabrication, and space qualification of AR microstructures for staring format $\mathrm{HgCdTe}$ FPA windows, is reported here. Transmission data for FPA windows containing AR microstructures is presented, demonstrating a reduction of reflected light loss from $21 \%$ for an untreated window down to an average of less than $1 \%$ over a six micron wide spectral range in the long wave infrared region $(7-13 \mu \mathrm{m})$. The potential for AR microstructures to perform over even wider bandwidths such as the important dual-band infrared region $(3-12 \mu \mathrm{m})$, has been demonstrated. Such high AR performance is coupled with nearly un-measurable scattered light losses as recorded by sensitive instruments operated by NIST. Initial proton radiation exposure and thermal cycling tests show no damage to the microstructures and no degradation of the AR performance. Interferometer measurements of the surface flatness of FPA windows incorporating AR microstructures indicate no change from the initial surface flatness, a result that is a significant improvement over thin-film AR coatings, and one that has great potential for large format FPA fabrication.
\end{abstract}

Keywords:Anti-Reflection, Motheye, Microstructures, Radiation-Hardened, Effective Index, HgCdTe, LWIR, Coatings

\section{INTRODUCTION}

The use of infrared (IR) light in military, industrial, space and commercial applications has expanded significantly in recent years. Imaging sensors, or focal plane arrays (FPAs), designed to detect the IR light signature of ballistic missiles, are a key component of the Missile Defense Agency's constellation of satellites known as the Space Tracking and Surveillance System, or STSS.

High performance sensors for the detection of infrared light within the mid(MWIR, 3 to $5 \mu \mathrm{m}$ ) and long-wave (LWIR, 8 to $14 \mu \mathrm{m}$ ) spectral regions, are fabricated with the ternary alloy mercury cadmium telluride, or $\mathrm{HgCdTe}$. HgCdTe detector arrays are commonly grown on the backside of $\mathrm{Cd}_{96} \mathrm{Zn}_{.04} \mathrm{Te}(\mathrm{CZT})$ windows. In large area staring FPAs used in space based surveillance systems, the $\mathrm{HgCdTe}$ detector array is flipchip bump bonded to a silicon readout chip and illuminated through the infrared transparent CZT window. A typical hybridized $\mathrm{HgCdTe}$ FPA is shown on the right. In the backside-illuminated configuration, the polished CZT window will reflect $21 \%$ of incident MWIR/LWIR light. Minimizing such reflections from the substrate backside is a critical process and functional issue, as such reflections will lower detector quantum

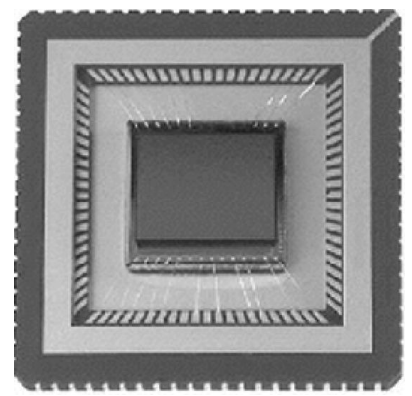
efficiency, increase detector noise and crosstalk, and result in poor image contrast.

*Correspondence: BDMacLeod@telaztec.com, Voice: 781-229-9906, Fax: 781-229-2195 www.telaztec.com

Infrared Technology and Applications XXXIV, edited by Bjørn F. Andresen, Gabor F. Fulop, Paul R. Norton, Proc. of SPIE Vol. 6940, 69400Y, (2008) · 0277-786X/08/\$18 · doi: 10.1117/12.778736 


\section{BACKGROUND - THIN-FILM ANTI-REFLECTION COATING TECHNOLOGY}

The conventional approach to suppressing reflections from CZT windows is to deposit multiple thin layers of dielectric materials. The film stack is designed to effect destructive interference over a limited IR wavelength band reflecting from the CZT window surface. Many thin-film layers are needed to increase the spectral range over which reflections are suppressed. Figure 1 shows the predicted reflection of LWIR light from an optimized 5-layer stack of thin films consisting of zinc sulfide and germanium deposited on a CZT substrate. The calculation was made using commercially available thin-film optimization program, and a cross section of the modeled structure is shown on the right. With five film layers the reflection of normally incident light is reduced to an average of about 3\% over an $8-12 \mu \mathrm{m}$ wavelength range within the LWIR. The effect of polarization splitting at large incident angles is also shown to illustrate the degradation in AR performance that can lead to

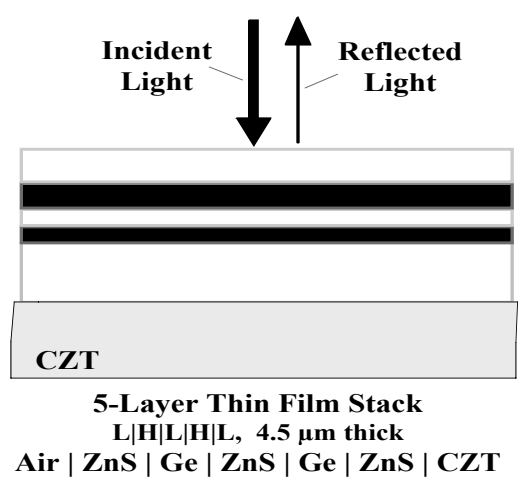
decreased FPA image contrast due to stray light reflections from the imaging lenses. Many STSS applications and related programs require less reflection loss over wider bandwidths, for example dual-band applications operating over the $3-12 \mu \mathrm{m}$ spectral range. Such performance requires many more thin film layers that increase the total stack thickness along with many of the stress related problems associated with deposited material coatings.

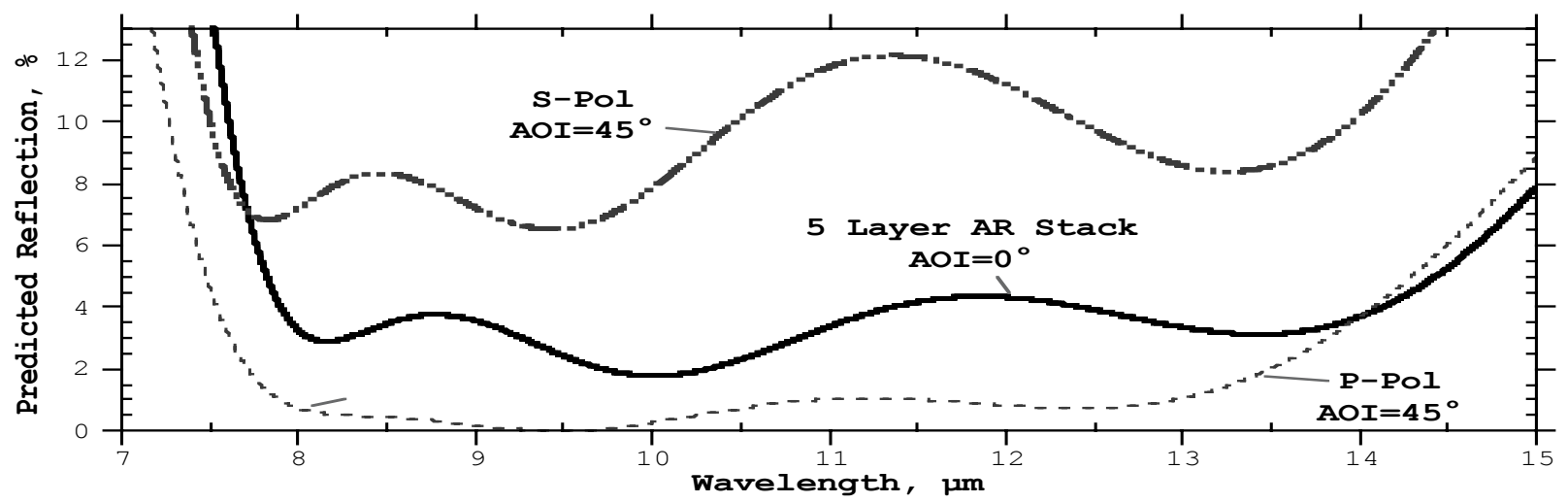

Figure 1: Predicted reflection of LWIR light from the thin-film coating stack depicted above.

Thin-film AR coatings are typically deposited by high temperature evaporation of the coating materials within a vacuum chamber, a process that is problematic for temperature sensitive materials such as $\mathrm{HgCdTe}$. At temperatures greater than $100 \mathrm{C}, \mathrm{Hg}$ can be driven from the crystal lattice creating parasitic electrically active $\mathrm{Hg}$ vacancies. As a result, the coatings must be deposited at temperatures lower than optimal for minimizing film stress and ensuring adequate thin film adhesion and density. This can result in durability and thermal stress issues at the low operational temperatures typical of space environments, due to the dissimilar thermal expansion coefficients of the thin-film materials and the substrate. Loss of adhesion and damage from temperature cycling can result in catastrophic failure of space-based IR imaging systems. Lastly, thin-film AR stacks are believed to suffer from degradation and decreased lifetimes in the presence of solar radiation - mainly high-energy protons.

\section{ARMS - MICROSTRUCTURE BASED ANTI-REFLECTION TECHNOLOGY}

AR Micro-Structures, or ARMS, have been shown to be an effective alternative to thin-film AR coatings in many infrared and visible-band applications ${ }^{[1-8]}$ where durability, radiation resistance, wide viewing angle, or broad-band performance are critical. These microstructures are fabricated directly into the surface of the window or optic material, imparting an optical function that minimizes reflections while maintaining the characteristics of the bulk material with respect to durability, thermal issues, and radiation resistance. The problems associated with thin-film coating adhesion, stress, and radiation hardness are removed by design; the thin films are eliminated.

TelAztec fabricates three distinct types of surface relief microstructures, commonly known as Motheye, SWS Effective Index, and Random Surface textures. Each type of structure has unique characteristics and optical properties that can be tailored for specific materials and applications. TelAztec has given detailed descriptions of the three structure types in the literature ${ }^{[9-12]}$, a brief outline of which is given here; 
1) MOTHEYE AR Structures: The Motheye structure is an array of surface depressions or pyramidal protrusions, such as those found on the eyes of nocturnal moths ${ }^{[1,2]}$. The tapered surface structure provides a gradual change of the refractive index for light propagating from air into the bulk optic material. Reflection losses are reduced to a minimum for broad-band light incident over a wide angular range. A typical Motheye texture profile is depicted below left where the height $\mathrm{h}$ and the spacing $\Lambda$ are indicated. A scanning electron microscope (SEM) image of Motheye structures fabricated in the surface of a CZT window, are shown on the right below.
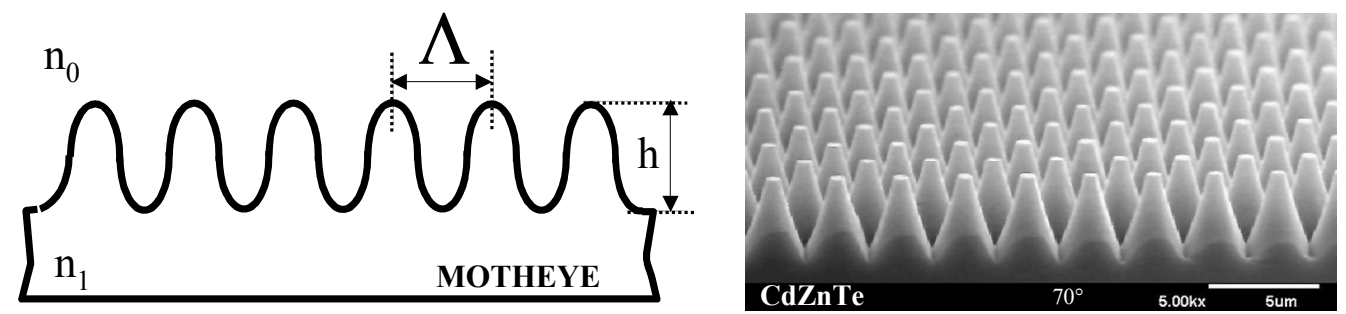

2) SWS Effective Index Structures: A Sub-Wavelength Structure, or "SWS" effective index texture is depicted in the profile diagram and SEM image below. An array of holes or posts provides an AR function that is equivalent to a single layer thin-film coating. The effective index is engineered to be the ideal index for the particular optic or window material, which is the square root of the material index of refraction. This is accomplished by tailoring the texture fill factor - the proportions of solid and open areas in the surface. Structure height $\mathrm{h}$ is then set to one quarter-wave optical thickness at the effective index. At one wavelength reflections are completely eliminated, and over a narrow wavelength band reflections are suppressed to very low levels. Dual and triple-band AR performance can be obtained with deeper structures set at multiples of the quarter-wave optical thickness.
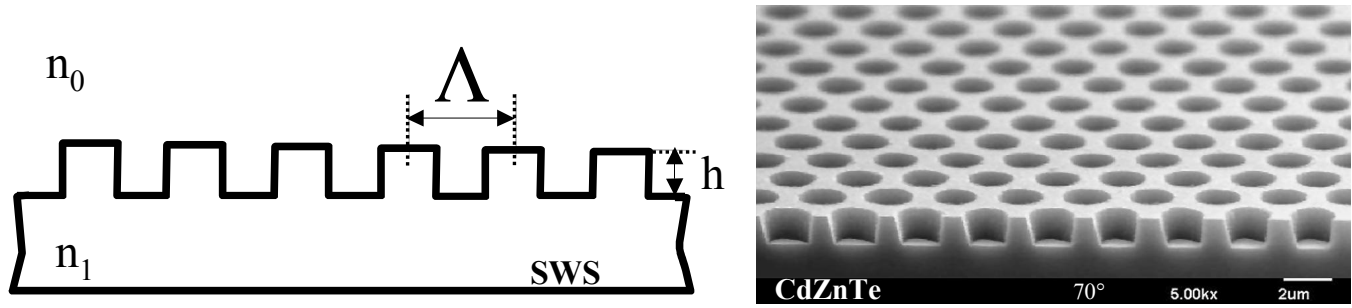

3) Random Texture AR Microstructures: TelAztec has developed a simple fabrication process for AR textures that have a random distribution of sub-wavelength sized surface features. The very small and dense features, as shown in the figures below, provide AR properties that are both extreme and broadband. Advantages of the Random AR texture include the cost driven benefit of eliminating the lithography step, and the ability to apply the structure conformal to challenging topologies, such as micro-lenses and MEMS. Random AR structures built in glass, plastic, and silicon windows exhibit extreme AR performance for visible and NIR light.
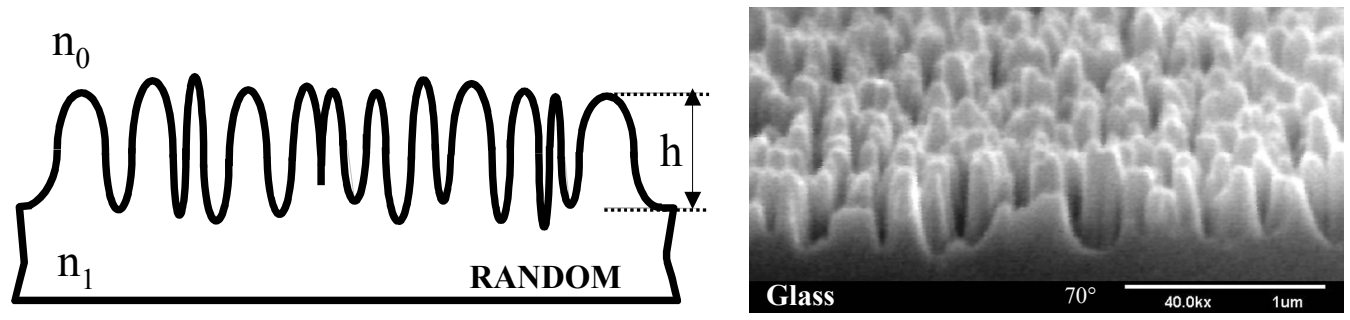

To achieve high performance AR with surface relief microstructures, optical phenomena such as diffraction and scattering must be avoided. This requires that the surface structures be fabricated with feature spacing $(\Lambda$ in the figures above) smaller than the shortest wavelength of operation within the material for a given application. In addition, for Motheye and Random AR structures, the height and cross sectional profile of the surface features must be sufficient to ensure a slowly varying density change. In general, AR microstructures will exhibit similar characteristics as the bulk material with respect to mechanical durability, thermal issues, laser power handling capacity, and radiation resistance. The problems with thin-film AR coating adhesion, stress, off-axis performance degradation, durability and lifetime, are eliminated. 


\subsection{ARMS MODELING}

TelAztec uses computer models to design and optimize the performance of Motheye and SWS AR microstructures. Using a rigorous vector diffraction calculation, the software can predict the spectral reflectance and transmittance of infrared light through a user defined three-dimensional surface texture. The model accounts for arbitrary polarization states and light incident angles. Modeled performance has proven to be an excellent match to the measured performance of fabricated AR optics when correlating with structural dimensions obtained from SEM. The ability to predict optical behavior and to optimize fabrication techniques is critical to the practical commercialization of the ARMS technology.

Transmission losses due to diffraction and scattered light are avoided by fabricating microstructures with dimensions that are less than the operational wavelength range within the material. As an example, for LWIR light that is normally incident upon a CZT window containing AR microstructures, the structure spacing, or period, is typically set below 3.0 microns $(8 \mu \mathrm{m} / 2.67)$, where the index of CZT is 2.67. TelAztec AR designs typically involve a periodic array of microstructures arranged in a hexagonal grid for maximum pattern symmetry and fill factor. In addition, the height of the structures is critical to the suppression of reflections at longer wavelengths. For Motheye and Random AR surface structures, the height is set to at least $40 \%$ of the longest design wavelength to realize the widest bandwidth performance. Next, TelAztec believes that AR microstructure mechanical durability will be optimum for structures with cross sectional profiles that do not contain sharp angles. Flat-top or truncated pyramidal and sinusoidal profile features are typically fabricated for most applications.

Modeling of the SWS structure, which operates on the principle of thin film interference, requires the feature depth to be set at a quarter wave optical thickness for the desired effective index and target wavelength. For example, to obtain peak AR performance in CZT at a wavelength of $10 \mu \mathrm{m}$, the feature height is $10 \mu \mathrm{m} / 4 / \mathrm{Sqrt}[2.67]$ or $1.53 \mu \mathrm{m}$.

The optical constants for cadmium telluride, CdTe are well known and available in the literature ${ }^{[13]}$. However, index of refraction data on $\mathrm{Cd}_{96} \mathrm{Zn}_{.04} \mathrm{Te}$ substrates for $\mathrm{HgCdTe}$ detector applications has not been reported. Spectroscopic ellipsometry was used for optical measurements of CZT to allow accurate ARMS modeling. A polished $1.0 \mathrm{~mm}$ thick (111) oriented CZT substrate was prepared with a $0.1 \%$ bromine in methanol etch for 20 seconds, followed by a methanol rinse. Measurements of the refractive index n, of both the Cd-rich (solid black line) and Te-rich (dashed black line) window faces, shown in Figure 2, agree well with the published literature for CdTe (solid gray line). Over a 3 to $24 \mu \mathrm{m}$ wavelength range, the absorption coefficient $\mathrm{k}$, was negligible, measuring less than 0.0001 . The $\mathrm{Zn}$ content in the measured substrate was confirmed by using Inductively Coupled Plasma Spectroscopy. The Cd to $\mathrm{Zn}$ ratio was found to be about 96.7/3.3, which is close to ideal for lattice matching to HgCdTe. These results show that the addition of a small amount of $\mathrm{Zn}$ to the CdTe structure does not appreciably alter the optical properties.

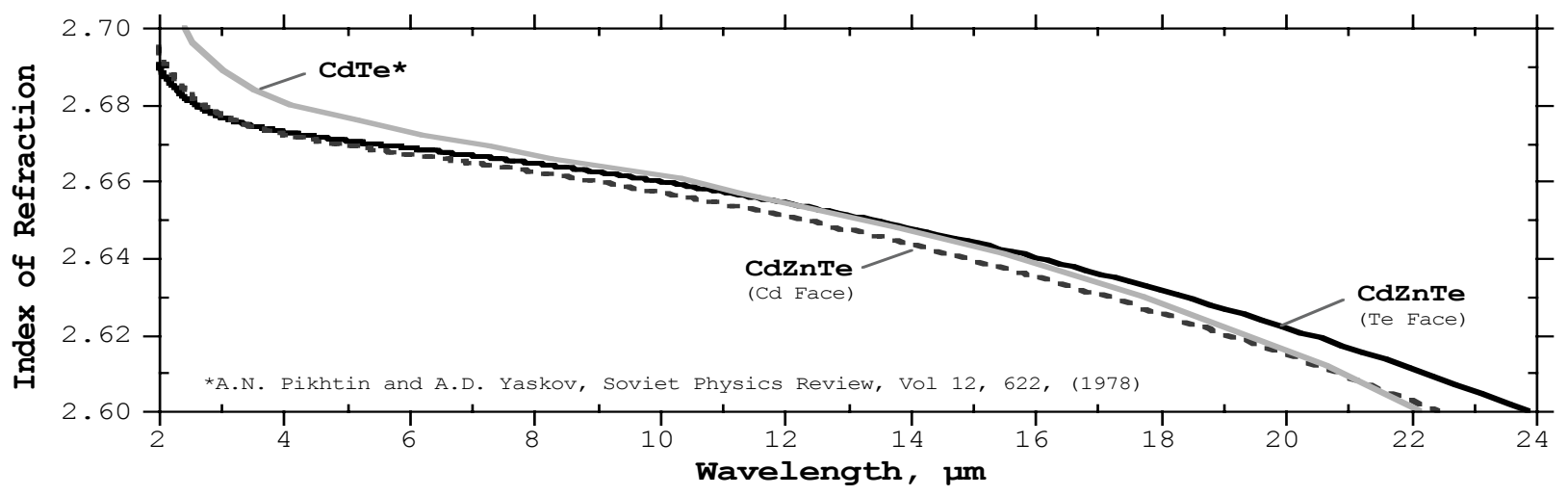

Figure 2. Measured refractive index of CdZnTe compared to CdTe published by Pikhtin and Yaskov.

\section{Motheye AR Textures in CdZnTe}

A model of the AR performance of various Motheye structure profiles in CZT designed for broadband operation over the 7 to $15 \mu \mathrm{m}$ wavelength band is shown in Figure 3. The graph shows the predicted transmission of infrared light propagating from air through various depth Motheye structures, and into a bulk CZT substrate (no backside effects). The Motheye structures were designed with a rugged flat-top triangular cross sectional profile. Note that once the Motheye structure depth reaches $4 \mu \mathrm{m}$, the predicted transmission exceeds $99 \%$ over the 7 to $14 \mu \mathrm{m}$ range, falling slightly to $98 \%$ at $15 \mu \mathrm{m}$. Similar performance for structures only $3.4 \mu \mathrm{m}$ deep is found when Motheye structures are fabricated with a flat-top sinusoidal profile. The parabolic shape in the Motheye texture valleys is often a desirable and 
natural outcome of some etching processes. Further modeling results for similar Motheye profiles operating on light incident at 30 degrees off-axis, show that reflectance losses remain below $2 \%$ over the $8-12 \mu \mathrm{m}$ spectral range.

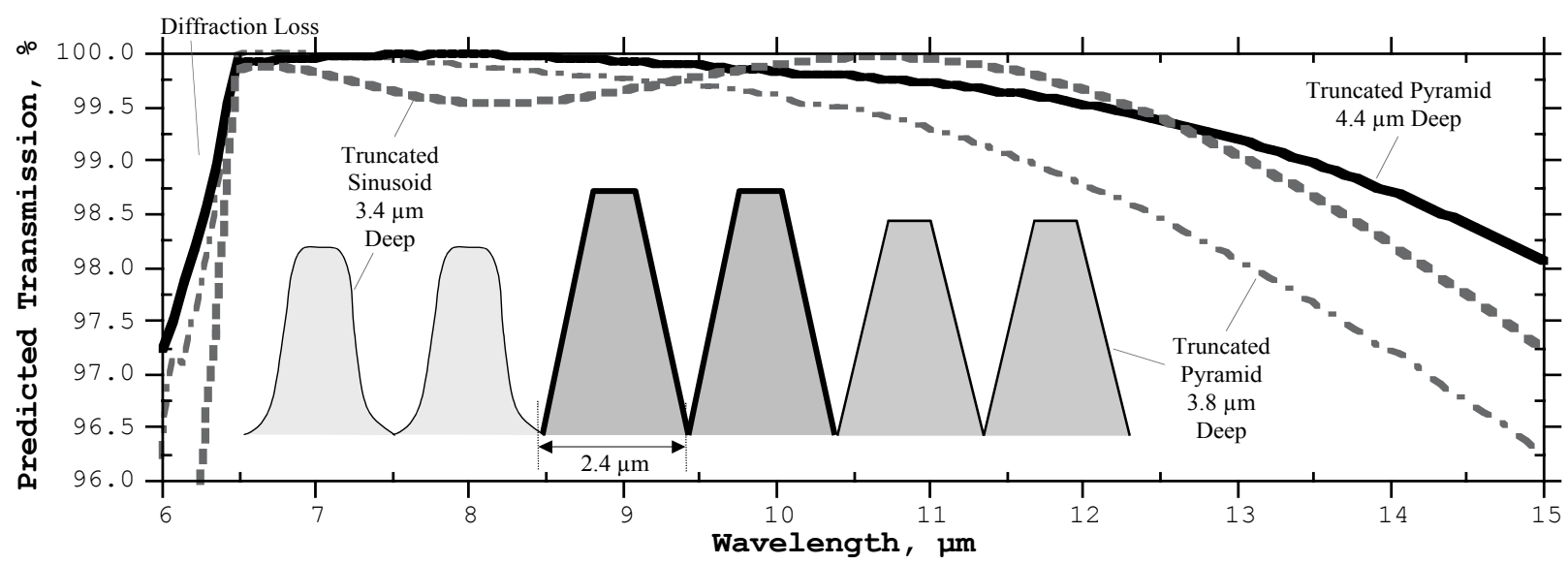

Figure 3. Predicted transmission of LWIR light through a Motheye structure fabricated into the surface of a CZT substrate.

\section{SWS Effective Index AR Structures in CdZnTe}

Modeling has been used to design and predict the performance of SWS Effective Index structures in CZT. Figure 4 shows an SWS structure with a predicted average reflection loss of less than one percent from 10-14 $\mu \mathrm{m}$, and effectively no loss at $12 \mu \mathrm{m}$. Performance at 30 degrees angle of incidence remains high with a peak performance shift toward shorter wavelengths as the effective "thickness" of the AR surface is reduced. The optimized fill fraction of 36\% CZT gives the ideal effective index of refraction at 1.63 , while the quarter wave etch depth is set to $1.8 \mu \mathrm{m}$. It should be noted that appropriate thin film materials with an ideal index are rarely available for specific applications. Another attractive aspect of the SWS texture is a simplified lithography and etch fabrication process.

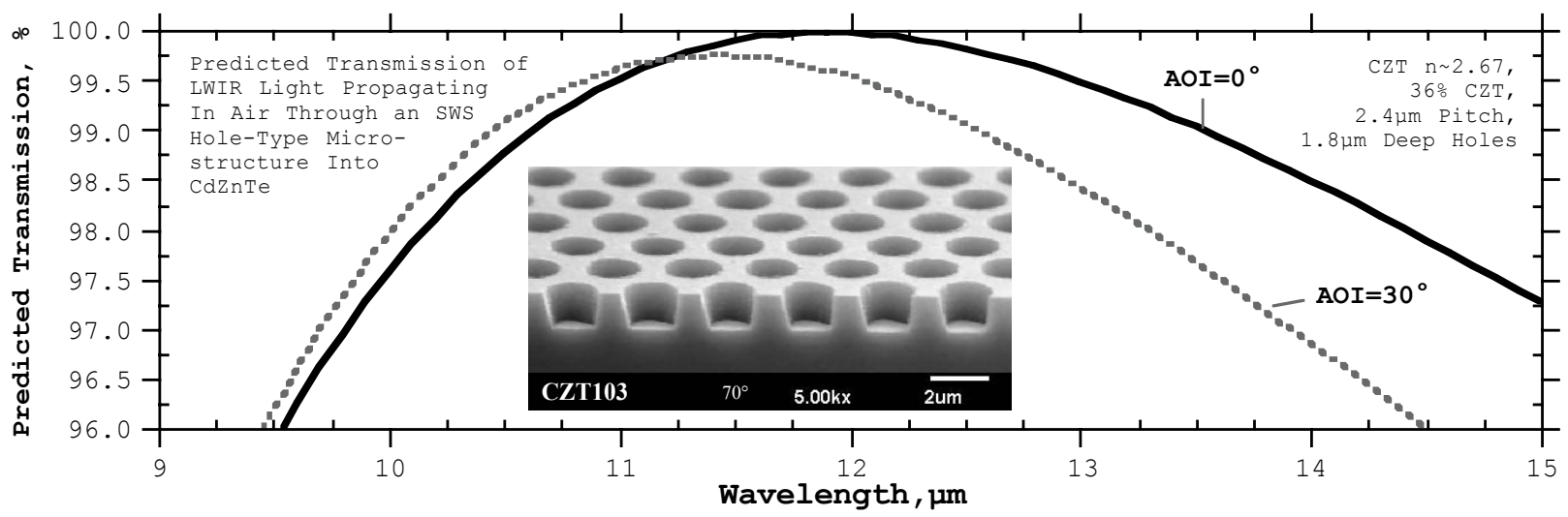

Figure 4. Predicted transmission of LWIR light through an SWS structure into CZT substrate.

The SWS structure described above is designed to maximize optical performance at a specified target wavelength. A novel dual band SWS structure can be designed by utilizing a harmonic of the effective index structure, which requires fabrication of a higher aspect ratio structure. For example, consider a binary structure in CZT with an effective index of 1.63, etched $1.60 \mu \mathrm{m}$ deep, but now with a period of $1.0 \mu \mathrm{m}$. The maximum transmission will be peaked at 3.7 and 10.8 $\mu \mathrm{m}$, providing some degree of dual band performance, as shown below in Figure 5. The thin film nature of this structure causes large reflectivity swings leading to an average reflection loss of $8 \%$ across the MWIR band, and an average $2 \%$ loss across the $8-12 \mu \mathrm{m}$ LWIR band. Such large swings in reflectivity can be minimized by incorporating a hybrid Motheye/SWS structure, yielding excellent dual band performance. The best performance structure modeled in Figure 5 depicts a $2.2 \mu \mathrm{m}$ deep tapered hybrid structure, which gives an average of less than $2 \%$ reflectance in both the MW and LW bands. 


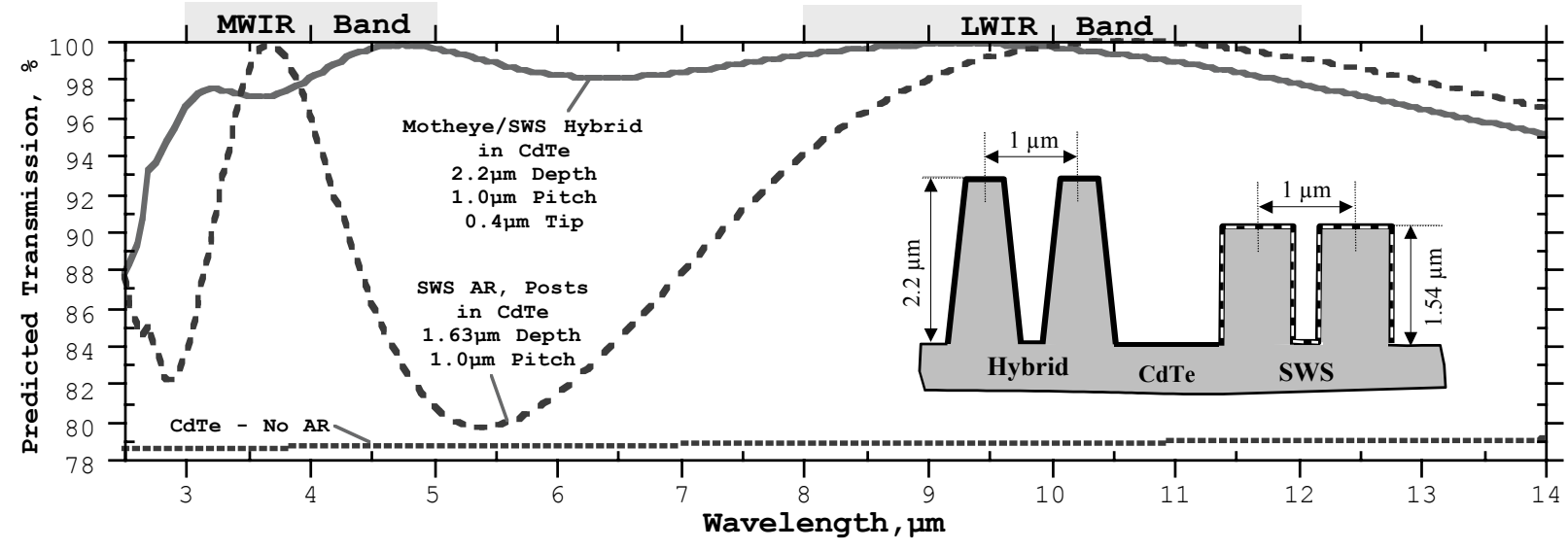

Figure 5. Predicted infrared transmission through a dual band SWS, and hybrid Motheye-SWS structure into CZT.

\section{ARMS TREATMENT - PROTOTYPE FABRICATION}

LWIR infrared transmitting materials such as CZT, require batch-processing methods to directly etch the AR structures into the window surface. Fabrication is a two-stage process consisting of a lithography step to pattern the microstructure in a sacrificial mask layer, followed by a conventional dry etching process to define the patterns in the surface of the final product. Because the sensitivity of $\mathrm{HgCdTe}$ devices to $\mathrm{Hg}$ vacancies, material defects, impurities, and handling damage is significant and of great concern, the introduction of innovative ideas to HgCdTe manufacturing is often met with resistance. The success of incorporating new device improvements is dependent on seamless integration into existing $\mathrm{HgCdTe}$ detector manufacturing processes.

After reviewing current $\mathrm{HgCdTe}$ device fabrication processes, it was determined that the optimal and least intrusive place to insert the ARMS process is at the wafer level - post Indium bump deposition and prior to wafer dicing. This is typically where thin-film AR coatings are deposited and allows for efficient wafer level ARMS processing. Figure 6 shows a process flow diagram typical of ARMS fabrication. The HgCdTe wafer device-side is protected with a polymer coating, and mounted device side down on a 4" diameter round carrier, such as alumina (step 1). The ARMS process begins with coating the CZT surface with a conventional positive photoresist (step 2). Next, a non-contact maskless lithography technique is employed to expose a latent image of the AR texture in the photoresist

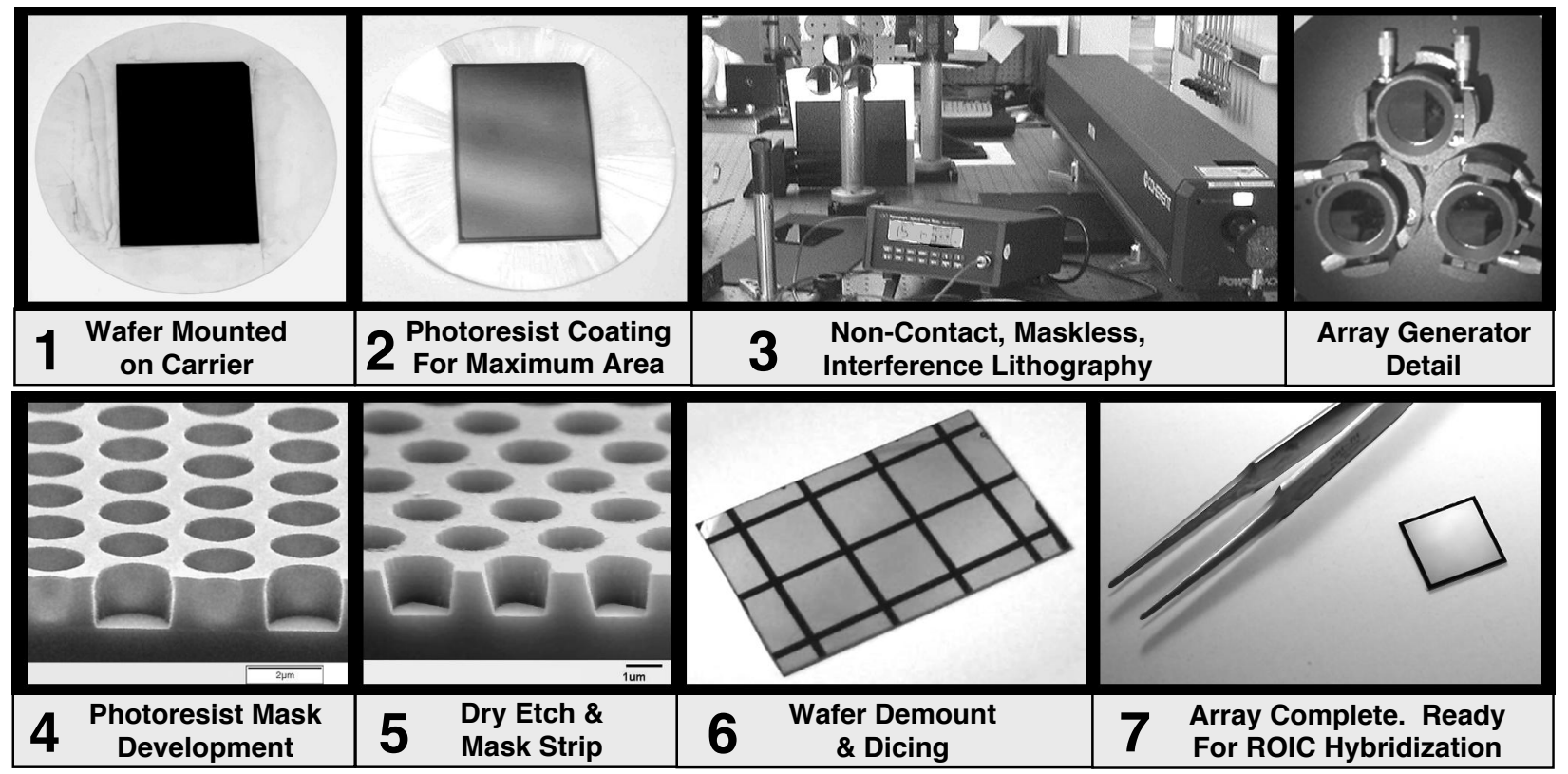

Figure 6: Wafer process flow diagram suggested for fabricating SWS ARMS in the backside of HgCdTe device windows. 
layer (step 3). The structure lithography is completed with a wet development step that delineates the image as a surface relief texture in the photoresist layer (step 4). The photoresist image is transferred into the CZT substrate with dry etching (step 5), with either Ion Beam Milling (IBM) or Reactive Ion Etching (RIE); and any residual photoresist is removed. If desired for process control, the performance of the AR microstructure can be measured in reflection while the sample remains mounted at the wafer level. The substrate backside with the ARMS is then protected with a spin on layer and diced through the backside, or the wafer is flip mounted for front-side dicing (step 6).

\subsection{HgCdTe- Special Process Considerations}

Photoresist Spinning: CZT substrates for $\mathrm{HgCdTe}$ are typically cut from the crystal boule in square or rectangular shapes, which poses unique challenges for photoresist spinning and subsequent coherent exposure lithography. While the $\mathrm{HgCdTe} / \mathrm{CZT}$ wafers are mounted on a round carrier, airflow during photoresist spinning creates turbulence near the corners of the CZT wafer, a phenomenon related to the Bernoulli Effect. This results in non-uniform photoresist thicknesses, particularly in the leading edges of the substrate corners. Non-uniform photoresist will cause exposure reflectivity variations and effective dose gradients across the wafer during exposure, resulting in poor feature size control. To address this problem, a covered spin chuck is used to minimize air turbulence in the spin bowl. The wafer shown in step 2 of Figure 6 exhibits a uniform photoresist layer and a consistent edge bead of less than $2 \mathrm{~mm}$.

Temperature Limitations Related to Hg: The CZT substrates that are used for $\mathrm{HgCdTe}$ detector growth have the challenging properties of being soft, brittle, and very expensive. The epitaxially grown layers of $\mathrm{HgCdTe}$ have the added issue of $\mathrm{Hg}$ volatility, forcing all process temperatures to be maintained below 90C. The ARMS fabrication process described above is compatible with this critical low temperature threshold. Substrate carrier mounting and photoresist baking processes are all maintained at or below 90C. Pattern transfer dry etch processes are kept at low temperature by utilizing cooled stages and thermal paste.

Wafer and Array Handling with ARMS: An approach to help with array level handling issues is to incorporate a non-textured street between the ARMS areas. By utilizing front to back side alignment methods, back side patterning of ARMS is confined to square areas corresponding to the front-side $\mathrm{HgCdTe}$ detector arrays. These square areas are delineated by "streets" without AR microstructures created by desensitizing the corresponding photoresist with a separate exposure step prior to the ARMS lithography step. At the completion of pattern development, etching, and photoresist removal, the streets remain as-polished next to the micro-textured areas. This design is expected to add a degree of post-dicing protection during tweezer handling and hybridization. Images of the diced array below demonstrate the edge street concept, with the Motheye structure recessed into the substrate.

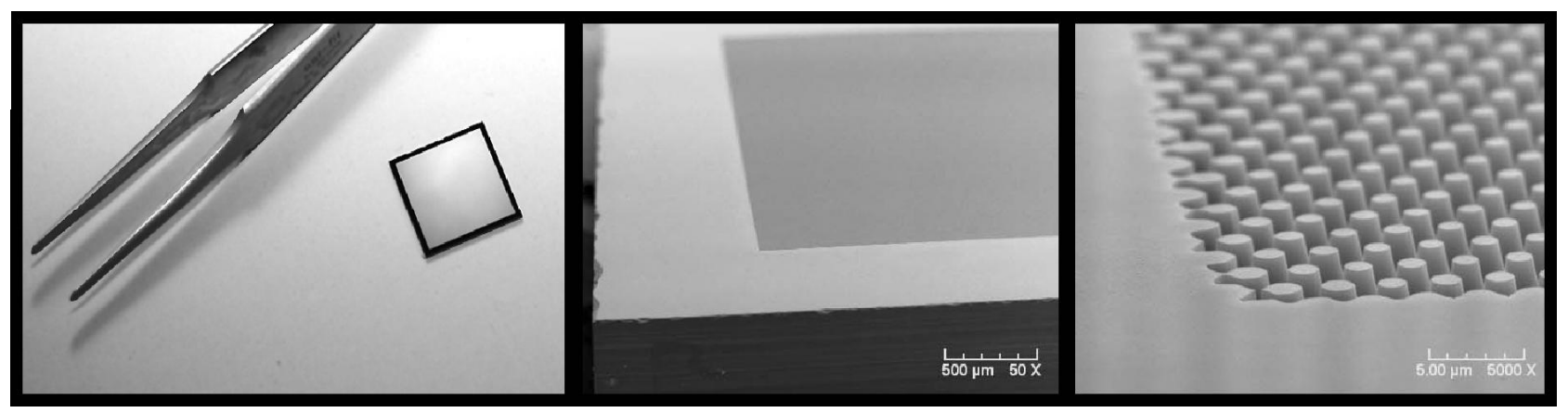

Diced CZT array and SEM images showing the edge street concept.

Hybridization with AR Microstructures: It is not believed that ARMS would be damaged by the high pressures employed for flip chip hybridization, due to the "bed of nails" effect of distributing pressure across the microstructure. However, debris or scratches on the hybridizer vacuum chuck could focus pressure locally and damage the textured surface. To keep the structures clean and pristine, the ARMS surface is coated with a protective layer of photoresist before the array is mounted on a carrier, device side up, for hybridization. This ensures an even distribution of pressure that prevents structure damage. At the completion of ROIC hybridization, the carrier and protective photoresist layer is soaked off of the FPA using solvents, and the hybrid is ready for packaging. 


\subsection{Interference Lithography}

TelAztec employs an advanced photolithographic technique known as interference lithography (IL) to record sub-micron and larger periodic patterns $^{[14-15]}$. A bench-top IL tool is shown on the right. Multiple beams of light are split from a laser source (typically emitting in the blue or violet), and expanded and redirected to overlap in a region of space where the resulting interference pattern can be recorded. The platform in the lower part of the photograph is illuminated by three overlapping beams derived from the laser source located at the back edge of the vibration isolation table. The three-beam configuration creates a high contrast, hexagonal grid periodic pattern that is ideal for fabricating AR microstructures. An IL system can pattern large field sizes, limited only by the size of the beam that is created, in a single rapid exposure. Highly uniform AR microstructures have been fabricated over 6-inch diameter windows. A significant advantage to IL patterning is a very large depth of field, on the order of inches, which eliminates depth of focus problems that spherical optics or non-flat substrates, can present to conventional lithography equipment such as image projection steppers and contact mask aligners. In addition, the maskless IL process eliminates any concerns of contact damage with delicate materials such as $\mathrm{HgCdTe} / \mathrm{CZT}$. Figure 7 shows SEM images of various periodic patterns recorded in photoresist using an IL system. The left side of the figure shows both elevation and overhead views of a photoresist mask containing hole-type structures defined on a CZT window intended for LWIR operation. The pattern has a hexagonal pitch of $2.3 \mu \mathrm{m}$ and a photoresist height of about $1700 \mathrm{~nm}$. Post-type structures in a photoresist mask defined on a CZT substrate are shown in the center area of the figure. The posts are about 1600 $\mathrm{nm}$ high and spaced on a $2.3 \mu \mathrm{m}$ grid. Lastly on the right side of the figure, post-type structures in resist on a sapphire window intended for MWIR AR applications are shown. These posts are about $1.5 \mu \mathrm{m}$ high with a hexagonal grid spacing of $1.6 \mu \mathrm{m}$.

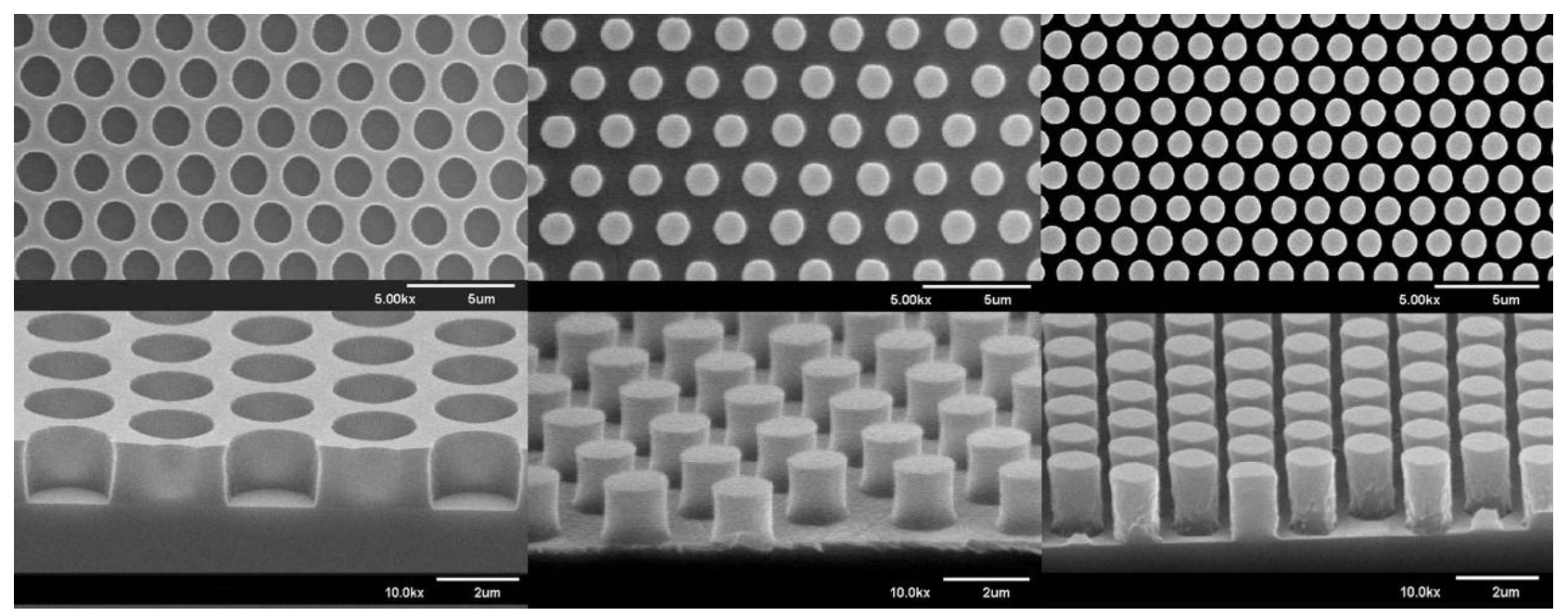

Figure 7: SEM micrographs of different periodic patterns defined in photoresist for use in the fabrication of ARMS.

The photoresist mask created by the IL tool is used to transfer the microstructure array into the surface of the substrate material using standard dry etching techniques. A thorough understanding of the interaction between lithography and the pattern transfer dry etching process is essential to fabricating ARMS, as the completed microstructure profile often bears little resemblance to the starting photoresist mask profile. This is because the physical and chemical aspects of etching are unique to each material, and process knowledge and control must take this into account. 


\section{ARMS TREATMENT - PERFORMANCE DATA}

Motheye and SWS AR textures were primarily fabricated on double side polished (111) oriented $\mathrm{C}_{.96} \mathrm{Zn}_{.04} \mathrm{Te}$ substrates. No differences in etch characteristics were noted for $\mathrm{Cd}$ vs Te face etching. Samples were characterized dimensionally using SEM analysis and optically using a Nicolet FTIR spectrometer to record the infrared transmission. In addition, precise microstructure pitch and symmetry was configured using diffraction measurements obtained with a fibercoupled white light spectrometer arranged in the Littrow configuration.

\section{Motheye Antireflection Surface Structures in CZT}

Dozens of CZT Motheye prototypes have been designed and fabricated to address STSS LWIR applications. Sample CZT58, which represents a typical LWIR Motheye CZT sample, was fabricated with the process described in Figure 6. The pyramidal structure has a hexagonal grid spacing of $2.3 \mu \mathrm{m}$ and structure height of $3.0 \mu \mathrm{m}$. The tapered surface structure, shown in Figure 8, was designed with a blunt tip profile for increased mechanical durability and is quite similar to the Motheye modeling designs depicted in Figure 3. Note that the flat tops of the structure represent the original polished CZT surface. Transmission measurements in Figure 9 show minimal loss of normally incident light at the Motheye surface, with an average reflection loss of 2\% across a broad 8-13 micron wavelength range in the LWIR, a significant improvement over the $21 \%$ loss for a polished surface with no AR treatment. The small difference between the "CdTe single surface theoretical" and CZT58 is signal loss that is not identified in this measurement, and is likely a combination of reflection, scatter, and absorption. The lithography and etching processes optimized for this effort have proven remarkably reproducible for Motheye surfaces in CZT.

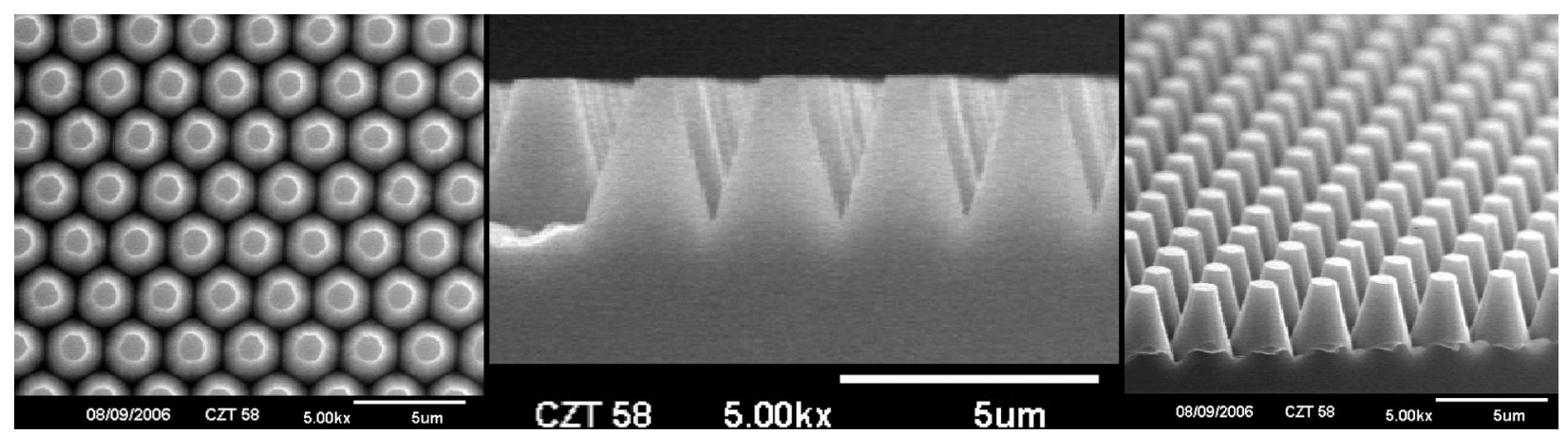

Figure 8. SEM images of a Motheye structure fabricated in a CZT window.

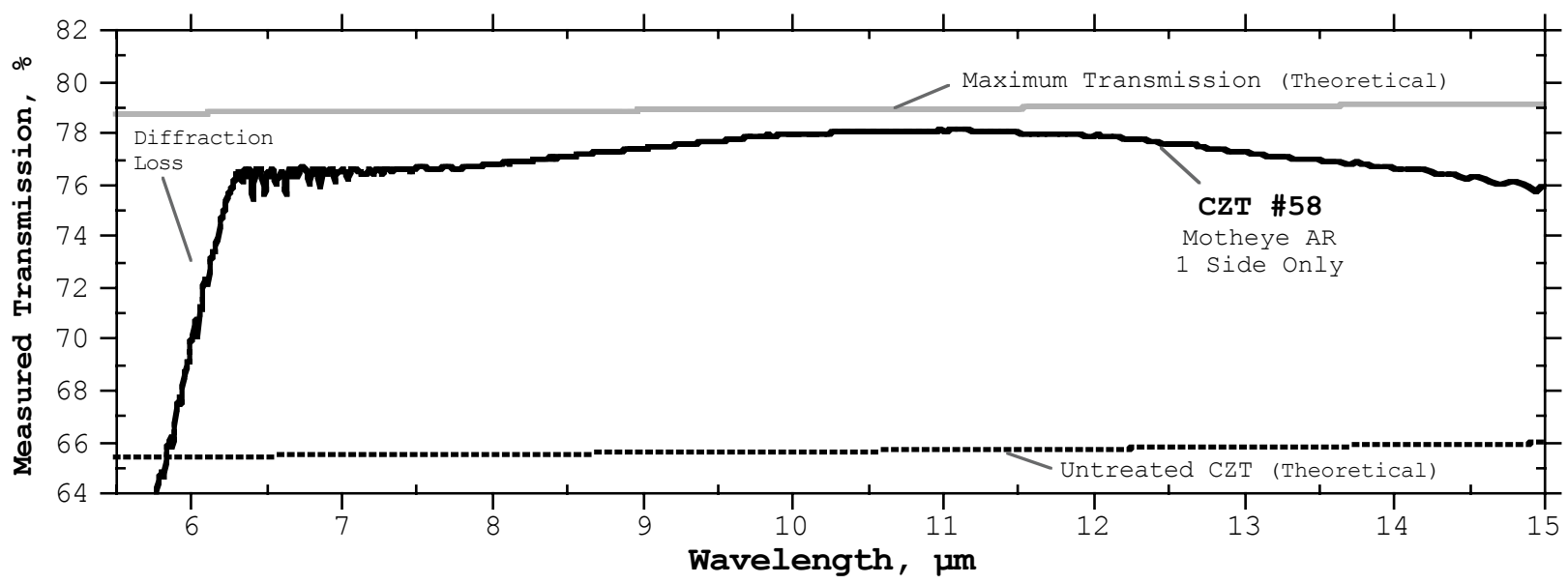

Figure 9. Measured transmission through CZT sample \#58 fabricated with a Motheye texture in one surface.

\section{SWS Effective Index Antireflection Structures in CZT}

Figure 10 shows SEM images of a SWS effective index AR structure fabricated in a CZT substrate and designed for operation in the LWIR region. The measured transmission through SWS-textured sample CZT 95J is shown in Figure 11. An average reflectance loss of less than $1 \%$ is observed for wavelengths ranging from 10 to $13 \mu \mathrm{m}$. 
The SWS ARMS process is based upon a high contrast photoresist pattern obtained with 3-beam IL (Figure 6), ensuring steep sidewalls in the subsequent anisotropic dry-etching process. Precision in the dry etching of SWS structures is more critical than with graded index Motheye structures due to the fact that the etch depth represents the QWOT and will center the performance peak accordingly. For example, a deeper etch depth for sample CZT 95J shown below, would shift the performance peak at $11.5 \mu \mathrm{m}$ to longer wavelengths, and the average performance over the 8-12 $\mu \mathrm{m}$ range would be reduced. Both SWS and Motheye ARMS can benefit from in-situ optical monitors for dry etching.

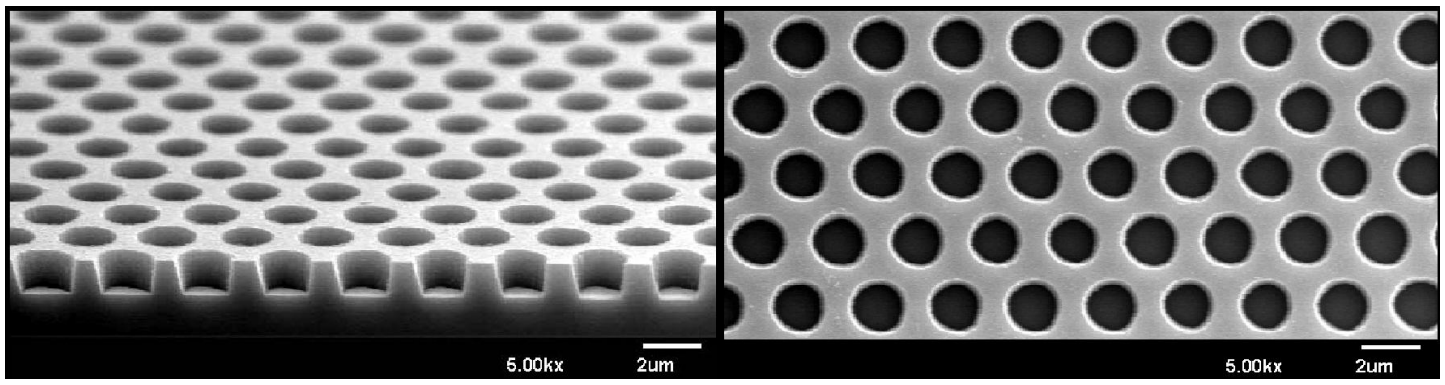

Figure 10: SEM images of an SWS effective index AR texture fabricated in a CZT window.

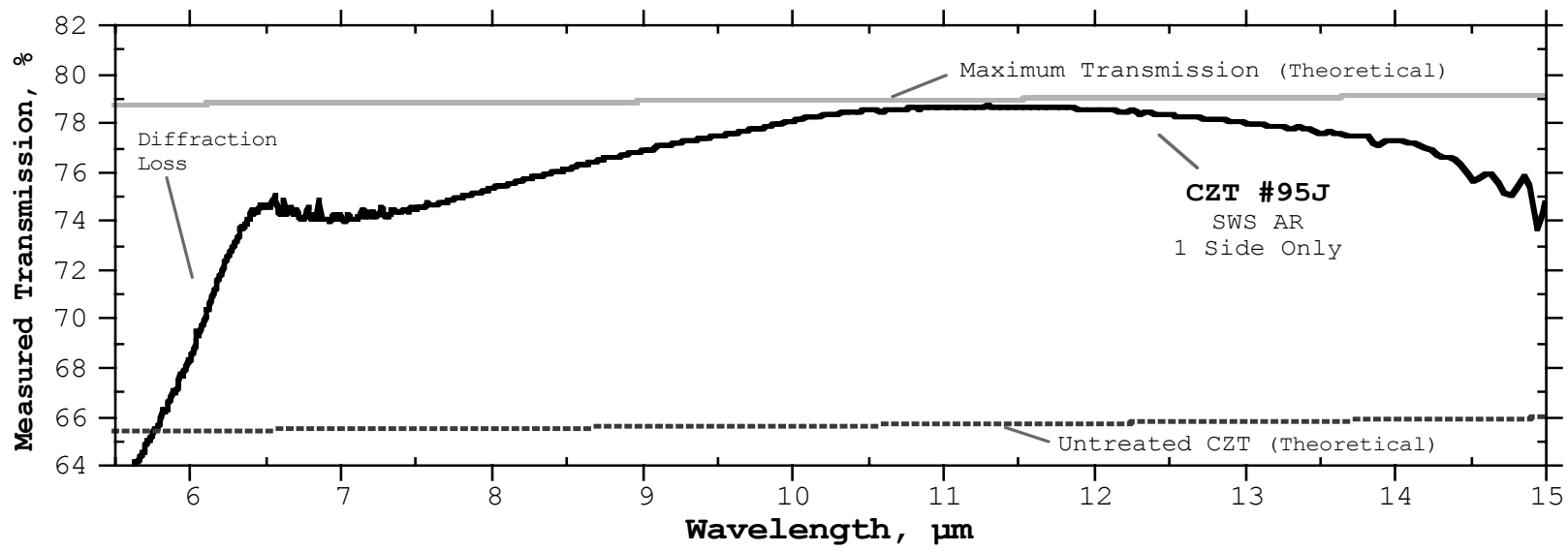

Figure 11: Measured transmission through a CZT substrate with a binary SWS AR texture fabricated in one surface.

\section{SWS Dual Band Effective Index Antireflection Structures}

A prototype dual band SWS "hybrid" structure designed for LWIR/VLWIR operation, was fabricated by etching a LWIR design SWS Effective Medium structure deeper and tapering the sidewalls. CZT 21 is shown in the SEM images of Figure 12, and has an etch depth of $3 \mu \mathrm{m}$, with broad flat tops and a flat base, and the slight taper characteristic of a hybrid structure. Figure 13 shows transmission data for CZT 21, where transmission peaks are found at 7 and $18 \mu \mathrm{m}$. The onset of free space diffraction loss below $7 \mu \mathrm{m}$ prevents adequate performance in the MWIR region with this design. Decreasing the pattern period from the $2.3 \mu \mathrm{m}$ period of the CZT21 sample to a spacing of $1.1 \mu \mathrm{m}$ would shift the diffraction edge below $3 \mu \mathrm{m}$ to yield a MWIR/LWIR dual band structure.

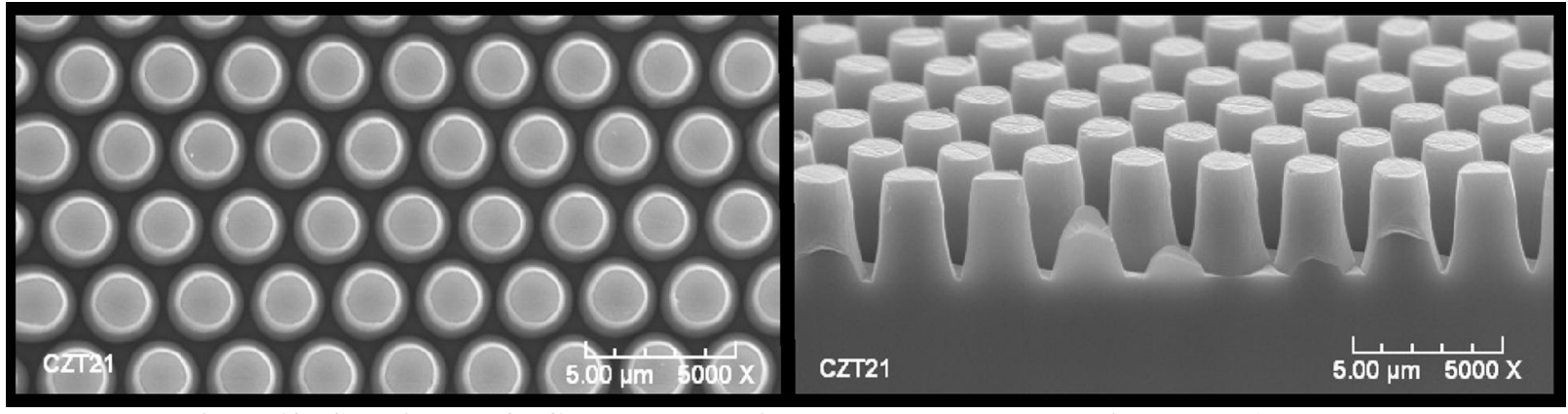

Figure 12: SEM image of a CZT substrate with a prototype dual band binary type AR texture 


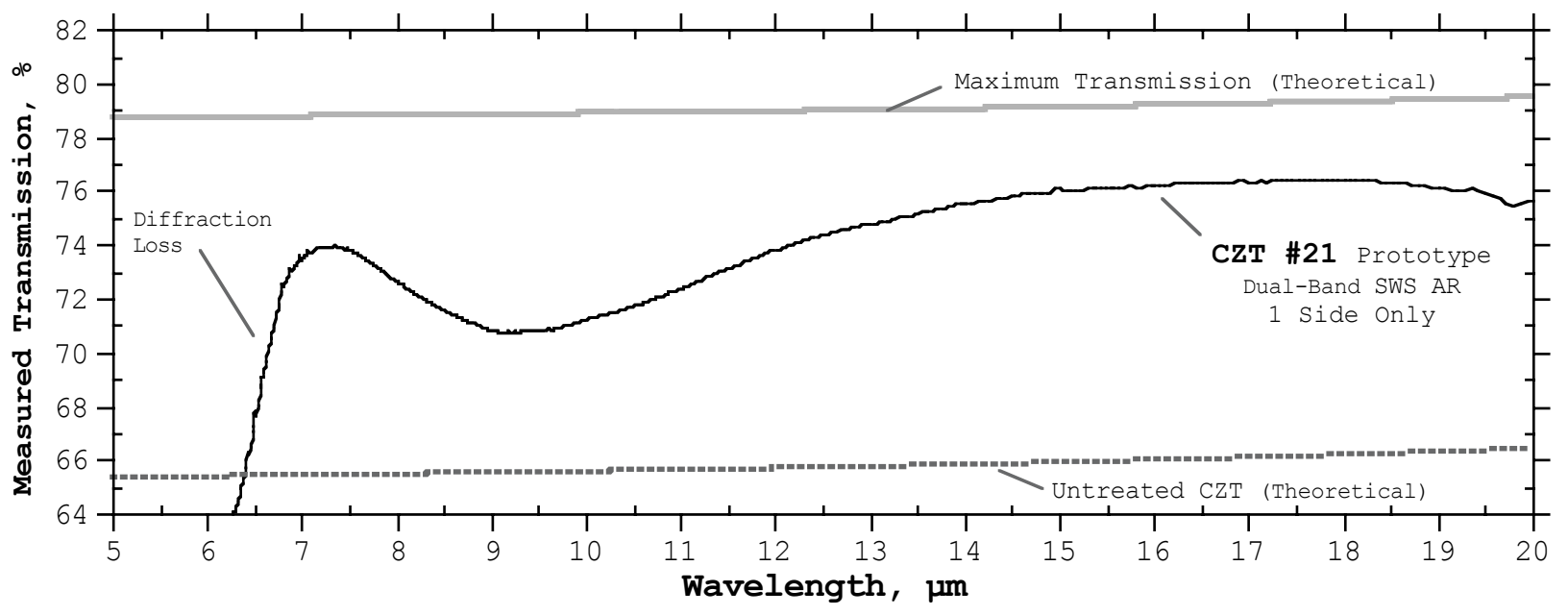

Figure 13: Measured transmission through a CZT substrate with a prototype dual band binary type AR texture

\section{Integrating Sphere Transmission and Reflection Measurements}

TelAztec's AR microstructures have been shown to give unparalleled broad-band optical performance. Motheye sample CZT58 as previously detailed in Figures 8 and 9, shows excellent performance, but with some minor transmission loss from 7-10 $\mu \mathrm{m}$ and $12-15 \mu \mathrm{m}$. The question that invariably arises from system engineers is "where does the rest of the light go?" There is concern that scattered light within the transparent substrate could create noise across the array, and degrade system performance. To address this concern, Motheye sample CZT58 was sent to the National Institute of Standards and Measurement (NIST), where the sample was measured for specular and diffuse components transmission and reflection. A Fourier Transform System using an Infrared Integrating Sphere ${ }^{[16]}$ configured with a $10 \mathrm{~mm}$ diameter beam size, was used to collect the light reflected and transmitted through the $16 \mathrm{~mm}$ x $16 \mathrm{~mm} \times 0.7 \mathrm{~mm}$ array sized sample. The "total" measurements of transmittance and reflectance are at 8 degrees incidence (f/6), directional-hemispherical. "Diffuse" results are at 0 degree incidence, Specular component excluded (f/4.5). The "Specular" results are obtained by subtraction. It is believed that there is no change in AR structure performance between 0 and 8 degree incidence for graded index structures based on ARMS modeling results. The 2 Sigma uncertainty is less than $0.3 \%$ for Specular transmission and reflection data, and $1.5-3.0 \%$ for Diffuse measurements, which is extreme precision for this type of measurement.

The plot in Figure 14 shows the NIST transmission measurement results. For clarification, Specular transmission (good) is defined as transmission unimpeded by the CZT substrate. Scatter or Diffuse transmission (bad) is defined as all other collected transmitted light within the integrating sphere. The Total transmission as measured by NIST, is the top trace in the plot corresponding to the left Y-axis, and represents the sum of Specular and Diffuse transmission. The Diffuse measurements of transmission and reflection are plotted against the right y-axis scale. The critical Specular transmission is obtained by subtracting the Diffuse transmission component from the Total measured transmission values. In a functional FPA, this is the image light that will be unimpeded by the CZT substrate and AR microstructure, and will be incident on the collection region of the correct detector pixel. Also shown on the plot is the "CdTe single surface theoretical" line, which represents a CdTe substrate with no reflection from one surface. Effectively, this is the theoretical maximum transmission that can be attained by AR treatments on one surface of a CdTe or CdZnTe substrate.

These results are dramatic. The critical Specular transmission data is actually $1 \%$ higher than we previously measured for CZT 58 from 7-12 $\mu \mathrm{m}$, based on our in-house FTIR spectrophotometer measurements. In the critical 8-12 $\mu \mathrm{m}$ LWIR band, specular transmission loss is well below an average of $1 \%$ (compared to CdTe single surface theoretical line). An average of $0.3 \%$ Total Diffuse loss is measured in the $8-12 \mu \mathrm{m}$ band, and represents no significant light scattering issues for noise in the FPA. It is not clear where the source of the minimal scatter is; it actually could be from inclusions or precipitates in the CZT substrate itself. As noted earlier, the beam size is approximately $10 \mathrm{~mm}$ diameter, so the sampled area covers the footprint of tens of thousands of pixels. 


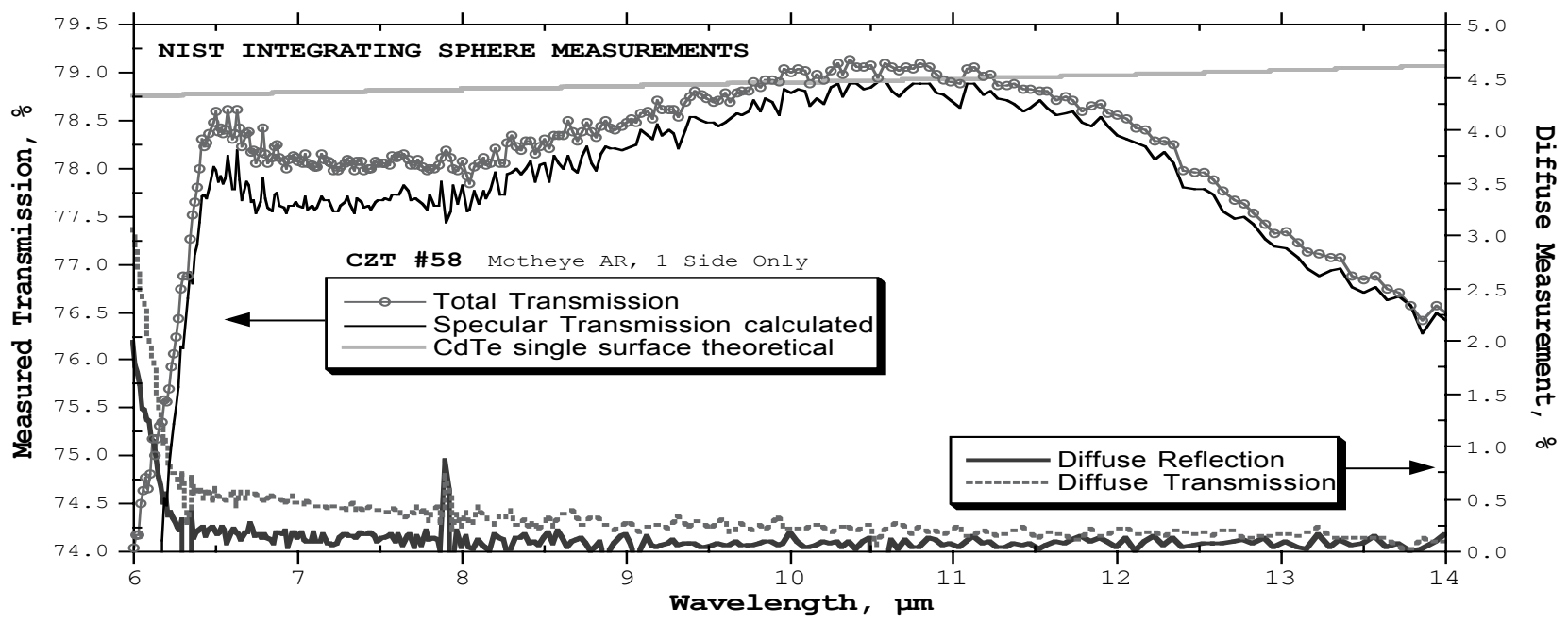

Figure 14. Specular and Diffuse measurements of Transmission and Reflection for Motheye sample CZT58

Figure 14 includes specular and diffuse transmission and diffuse reflection, but does not include specular reflection due to scale constraints. Note that the Specular reflection, which is around $21 \%$, is primarily from the untreated side of the substrate, and not a concern in a backside illuminated FPA configuration. For an ideal Integrating Sphere measurement, the sum total of all transmission and reflection data should equal $100 \%$. Figure 15 shows the sum total of measured light and compares it to the $100 \%$ line representing the light input into the Integrating Sphere. From $6.5-12.0 \mu \mathrm{m}$, $99.7 \%$ of the incident light is collected and identified. From $12-14 \mu \mathrm{m}$, the loss increases, possibly due to substrate absorption or light unmeasured due to wave guiding within the CZT substrate.

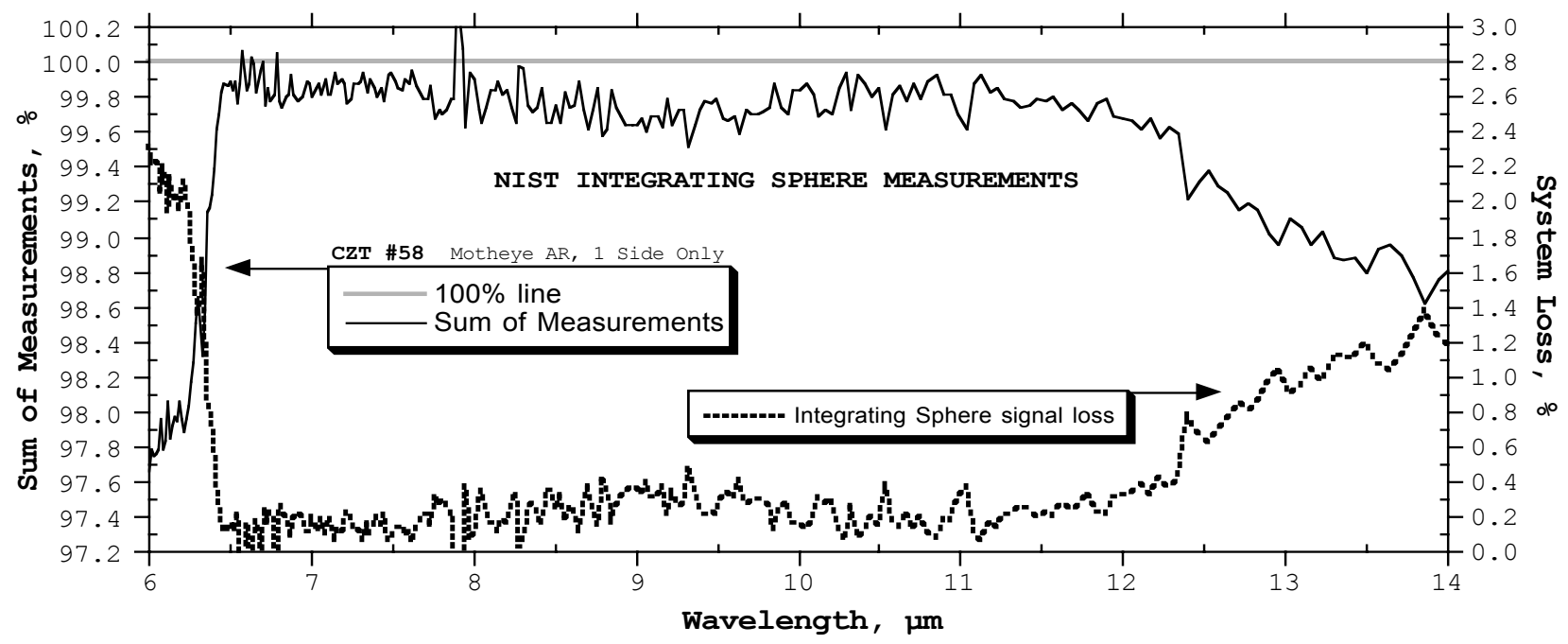

Figure 15. Total measured light, and signal loss for CZT 58 measurement

In summary, we can say the following about the AR performance of CZT 58 in the 8-12 $\mu \mathrm{m}$ LWIR band: On average, we have $21.1 \%$ reflection off the substrate backside (expected), $0.5 \%$ specular reflection loss off the Motheye surface, $0.4 \%$ light scattered diffusely (both transmission and reflection), $0.3 \%$ lost to the measurement system, and specular transmission of $77.7 \%$. Because the substrate backside, which is reflecting $21.1 \%$, is representative of the $\mathrm{HgCdTe}$ absorbing layer in an FPA, the conclusion is that over 98\% of the incoming LWIR 8-12 $\mu$ m light passes unimpeded to the HgCdTe collection region. To our knowledge, these measurements are the most comprehensive reported for AR microstructures and should eliminate any concerns about light interaction and scattering from properly designed and fabricated AR microstructures. 


\section{DURABILITY OF ANTIREFLECTION SURFACE STRUCTURES}

Thermal Cycling: The inherent durability of an AR surface etched directly into the substrate has great promise for providing long lifetime performance in the low operating temperatures of STSS imaging systems. The single material composition of ARMS textures is in stark contrast to the multiple material layers with multiple thermal expansion properties that are inherent with thin-film AR coatings. The operational requirement to cool $\mathrm{HgCdTe}$ detectors to cryogenic temperatures and below should have the same effect on the structure as it does on the bulk substrate, effectively resulting in no damage. Thermal shock tests on prototype ARMS arrays in CZT substrates confirm this result. Both Motheye and SWS samples were put through five cycles of aggressive immersion in liquid nitrogen followed by immediate heating to $370 \mathrm{~K}$. Subsequent sample inspection showed no damage to the surface structure and no degradation of the AR performance. Additional tests to $40 \mathrm{~K}$ are planned with an automated cycling system.

Radiation Hardness: In the extreme radiation environment of space, ARMS etched into the substrate should exhibit the same excellent resistance to impact with high-energy protons, and from damage due to solar gamma rays, as the CZT substrate itself. Any substrate damage or darkening from radiation exposure should be a bulk effect and should not be related to a surface treatment. Preliminary proton total dose irradiation (63.3 $\mathrm{MeV}$ protons) of CZT samples was completed by John Hubbs (AFRL Kirtland) at the Crocker Nuclear Lab (UC Davis). Array sized samples of CZT substrates treated with SWS and Motheye AR textures were mounted in a custom delrin plastic holder (shown on the right)

Mounted CZT substrates ready for radiation exposure along with samples of untreated and thin-film AR coated CZT. The matrix of 24 samples was irradiated at six total dose levels up to $1000 \mathrm{KRad}(\mathrm{Si})$. There was no evidence of mechanical or optical damage, as shown in Figure 16. However, radiation-induced damage is more likely seen in-situ at the low FPA operational temperatures. Future radiation tests are planned to include $40 \mathrm{~K}$ in-situ optical testing of AR microstructures in CZT, to eliminate the chance that radiation effects are annealed out at elevated temperatures.

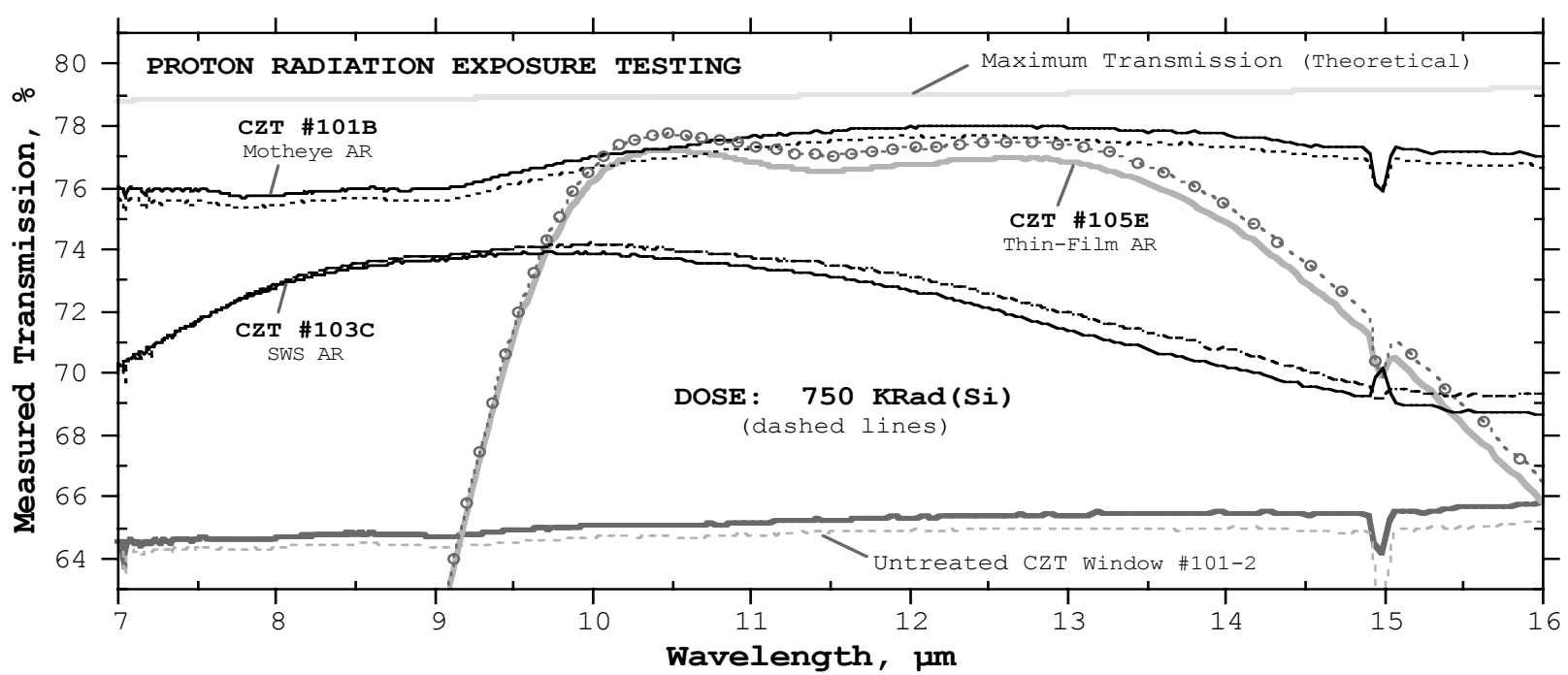

Figure 16: Transmission of thin-film AR coated, ARMS, and untreated CZT windows before and after radiation exposure.

Thin Film Coating Specifications: Thin film antireflection coatings have been tested for adhesion and abrasion under military specifications such as MIL-C-48497A. The specific tests described are not necessarily applicable to AR microstructures for the STSS application. In particular, the space based imaging application of this work is not abrasive by definition. The hybridized arrays are packaged and sealed in an evacuated dewar, where there are no moving parts, so there is no operational risk to abrasion damage. The concern with mechanical damage primarily lies in processing where carrier mount processes are used to protect the ARMS surface, particularly during hybridization. HgCdTe process engineers are well versed in carrier mount procedures for the brittle and expensive device wafers; and carrier mount processes are standard through the industry for large wafer $\mathrm{HgCdTe}$ on CZT device processing. 
ARMS samples do not have films to delaminate, however a few standard tape pull tests were performed on CZT Motheye and SWS samples using specified pressure sensitive tape CID-A-A-113B. The samples were waxed onto a carrier and the tape was pressed firmly onto the exposed micro-structured surface of the samples, then quickly pulled off at an angle normal to the surface. No physical damage was observed on either Motheye or SWS samples. Note that it is quite easy to see damage/defects under low magnification in a repetitive field of micron-sized features.

Abrasion tests for thin films specify protocol for rubbing samples with cheesecloth or an eraser. AR microstructures in CZT are expected to fail in these particular tests, partly due to the surface relief of the structure, and partly due to the CZT substrate itself likely failing both tests due to its soft characteristic.

While fabrication processes can be designed to eliminate ARMS surface structure damage during manufacture, it is worth discussing the effect of damaged or broken Motheye tips. A broken tip simply acts as a less ideal AR structure, for example, a 3.0 micron deep structure that loses the top 1.0 micron of the structure will still provide the good AR performance of a 2.0 micron deep structure with a non-ideal taper. This compares favorably with the effect of mechanical damage on AR coatings. A delaminated or damaged thin film coating can actually increase reflectivity and scattered light levels over an untreated surface, leading to FPA failure.

Stress Effects: The undesired stress effects of using dissimilar materials for multilayer thin film AR coatings are well documented. For space-based applications, where system requirements may require active detector cooling as low as $40 \mathrm{~K}$, the problems related to thermal coefficient of expansion of the different thin film materials and the substrate are exacerbated. The beneficial low stress nature of AR microstructures has not been previously reported.

To examine and compare potential stresses induced by AR coatings and ARMS, the surface flatness of a matrix of CZT samples was measured with a Zygo New View 5000 white light interferometer system. A set of 24 samples included 6 samples each of: CZT substrates (control), CZT with a conventional 5-layer LWIR AR coating stack, CZT with Motheye, and CZT with SWS Effective Index structures. All samples were nominal medium format staring array dimensions at $16 \mathrm{~mm} \times 16 \mathrm{~mm} \times 0.7 \mathrm{~mm}$, and measurements were performed at room temperature. The sampling area was $13 \mathrm{~mm} \times 13 \mathrm{~mm}$ centered on the backside of array, relative to the AR treatment. Figure 17 shows the average peak to valley (P-V) data of diagonal corner-to-corner measurements taken from the 3-dimensional topographical maps produced by the measurement system, as well as representative plots of the AR coated and Motheye samples. The surface flatness results are dramatic and show a P-V measurement of the thin-film AR coated samples of $2.05 \mu \mathrm{m}$, a value seven times larger than the AR micro-structured Motheye and SWS samples. The thin-film AR coated sample data indicates a concave surface figure due to a compressive stress on the substrate at room temperature. The ARMS samples themselves show a minimal flatness variation of $0.27 \mu \mathrm{m}$ that is comparable to the CZT substrate controls, and which appears unchanged from the initial substrate surface figure after polishing.
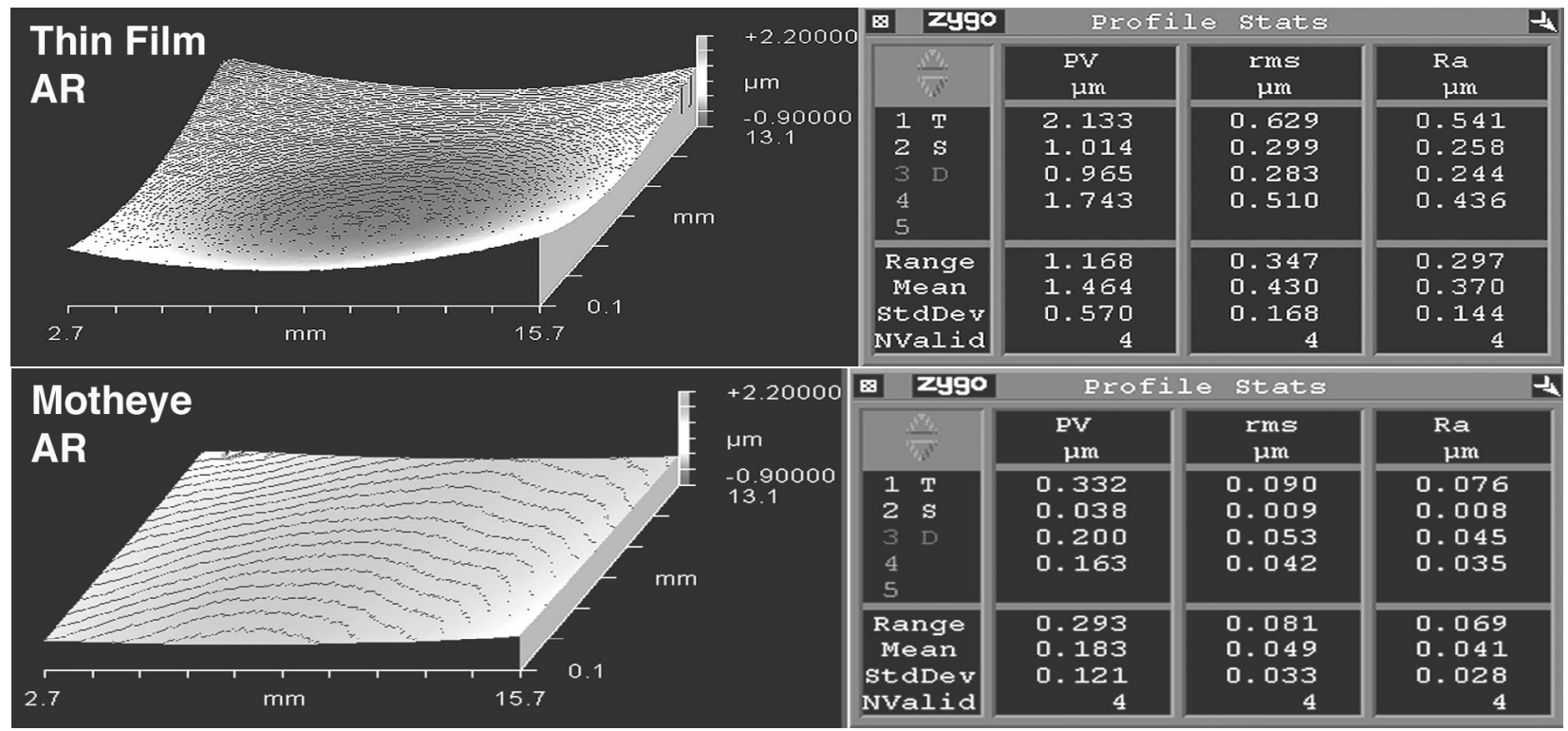

Ave. CZT substrate P-V $(\mu \mathrm{m}): \quad$ Untreated- 0.44; $\quad$ Motheye- 0.21； SWS- 0.33; $\quad$ AR Coating- 2.05

Figure 17 Zygo interferometer measurements.

Upper left shows the surface figure of a typical AR coated CZT sample, lower left shows CZT with Motheye AR structure. 
The results confirm AR microstructures incorporate no added stress to the optical component, in contrast to thin-film AR coatings. This conclusion is particularly relevant as application requirements increasingly demand larger format staring FPAs. It should also be emphasized that the stress seen in thin film AR coatings is greatly magnified at the low system operational temperatures typical of space-based sensors.

\section{SUMMARY}

The incorporation of surface relief microstructure arrays for anti-reflection in space-based infrared sensors that offer extreme optical performance, radiation hardness and environmental durability, has been demonstrated. Theoretical models were presented that illustrate the performance benefits of AR microstructures for various imaging applications.

Motheye graded index AR microstructures were fabricated in CZT windows used for LWIR HgCdTe infrared detectors. An average transmission loss of less than 1\% over the LWIR range for Motheye AR textured windows exceeds the performance of the best commercially available thin-film AR coatings. Similar extreme AR performance is reported for SWS effective medium AR microstructures fabricated in CZT windows. Such extreme AR performance is combined with nearly un-measurable scattered light losses as measured by sensitive instruments built and operated by NIST.

A novel hybrid SWS AR microstructure design was introduced and prototyped. The prototype exhibits broadband AR functionality, with performance peaking at multiple wavelengths, a significant potential benefit for MWIR/LWIR dual band applications.

The fabrication process for ARMS was detailed with an emphasis on the careful handling needed for damagefree processing of the delicate $\mathrm{HgCdTe}$ devices. A non-invasive method for integrating the ARMS fabrication process into existing $\mathrm{HgCdTe}$ process lines was discussed.

Interferometer surface flatness measurements were completed on ARMS and AR coated CZT windows. Results confirm that Motheye and SWS AR textures do not introduce any stress to the window, in stark contrast to the large compressive stress measured with the thin film AR coated samples. It is noted that problems related to thermal stress become magnified at the low temperatures of FPAs operating in space.

Full scale ARMS prototypes show excellent durability in preliminary thermal cycling and proton radiation exposure trials. The AR microstructures are expected to be as durable as the CZT window when exposed to the radiation levels found in space. Additional work is ongoing to confirm that HgCdTe FPAs incorporating ARMS and radiation-hardened ROICs will improve operational and lifetime performance of STSS imaging systems.

The ARMS modeling and development work described here is applicable to virtually all appropriate materials and infrared wavelengths. Previous TelAztec work has validated the ARMS concept in other infrared materials such as $\mathrm{ZnS}, \mathrm{ZnSe}, \mathrm{Ge}, \mathrm{GaAs}, \mathrm{ZnGeP}, \mathrm{As}_{2} \mathrm{~S}_{3}$, silicon, sapphire, and fused silica. ${ }^{[10-12[}$ The increased demand of advanced imaging systems for larger format arrays, increased sensitivity and wider bandwidth, lower operational temperatures, and increased durability make Anti-Reflective MicroStructures (ARMS) an attractive alternative over current thin film AR technology.

\section{ACKNOWLEDGEMENTS}

Much of the work with CZT AR surface structures has been funded by a 2005 Phase I SBIR, and an on-going Phase II SBIR contract (W9113M-06-C-0155) sponsored by the Department of Defense, Missile Defense Agency, managed through the Materials and Manufacturing Directorate, Air Force Research Laboratory, Wright Patterson Air Force Base (see approval notice below). A great amount of advice and guidance on the needs and requirements of the STSS program has been provided by L. Kevin Slimak of the MDA's Space Sensors Division, and Robert J. Ondercin of AFRL's Materials and Manufacturing Directorate. Radiation testing was performed courtesy of Dr. John Hubbs, AFRL, Kirtland AFB. Helpful discussions on radiation effects were provided by Jonathan Fisher of GH Systems. Comprehensive optical measurements were provided courtesy of Leonard Hanssen of NIST. Cooperative development work was completed with IR detector manufacturers, specifically Paul Lovecchio, John Marcinec, and Themis Parados at BAE Imaging Systems, and Scotty Gilmore at Raytheon Vision Systems. Spectroscopic Ellipsometry was completed by J. A. Woollam Inc. Surface flatness measurements were taken by Zygo Corporation. All SEM analysis was performed by Mr. John Knowles at MicroVision Laboratories, Inc.

APPROVED FOR PUBLIC RELEASE: 07-MDA-3036 (18 DEC 07) 


\section{REFERENCES}

[1] Bernhard, C. G., "Structural and functional adaptation in a visual system", Endeavour, 26, pgs. 79-84, 1967

[2] Clapham, P.B. and Hutley, M.C., "Reduction of lens reflexion by the 'Moth Eye' principle", Nature, 244, 281-2, Aug. 3, 1973)

[3] Thornton, B.S., "Limit of moth's eye principle and other impedance-matching corrugations for solar-absorber design." JOSA, Vol. 65, No. 3, pgs 267-270, March 1975

[4] Wilson, S.J. \& Hutley, M.C., "The optical properties of 'moth eye' antireflection surfaces", Optica Acta, Vol. 29, No. 7, pgs 993-1009, 1982

[5] Southwell, W. H., "Pyramid-array surface-relief structures producing antireflection index matching on optical surfaces", JOSA A, Vol. 8, No. 3, pgs 549-553, March 1991

[6] Raguin, D.H. \& Morris, G.M., "Antireflection structured surfaces for the infrared spectral region", Applied Optics, Vol. 32, No.7, pg 1154, March 1993

[7] DeNatale, J. F., et. al., "Fabrication and characterization of diamond moth eye antireflective surfaces on Ge", J. Appl. Phys.,71, (3), pg1388,Feb.1992

[8] Harker, A.B. and DeNatale, J.F., "Diamond gradient index 'moth-eye' antireflection surfaces for LWIR windows.", SPIE Vol. 1760, Window and Dome Technologies and Materials III, pgs. 261-267, July 1992

[9] MacLeod, B.D., and Hobbs, D.S., "Low-Cost Anti-Reflection Technology For Automobile Displays", Journal of the Society for Information Display, Automotive Display Conference, November 2004.

[10] Hobbs, D.S., and MacLeod, B.D., "Design, Fabrication and Measured Performance of Anti-Reflecting Surface Textures in Infrared Transmitting Materials", Proc. SPIE Vol. 5786, May 2005.

[11] Hobbs, D.S., and MacLeod, B.D., "Update on the Development of High Performance Anti-Reflecting Surface Relief Micro-Structures", Proc. SPIE Vol. 6545, April 2007.

[12] Hobbs, D.S., and MacLeod, B.D., "High Laser Damage Threshold Surface Relief Micro-Structures for AntiReflection Applications", Proc. SPIE Vol. 6720, Sept. 2007, (Boulder Damage: Received Best Paper Award).

[13] Pikhtin, A.N., and Yaskov, A.D., “ “, Soviet Phys. Semi., Vol.12, 622, 1978

[14] Hobbs, D.S., et. al., "Automated Interference Lithography Systems for Generation of Sub-Micron Feature Size Patterns", SPIE Conference on Micromachine Technology for Diffractive and Holographic Optics, Proc. SPIE, Vol. 3879, September 1999, pg 124-136

[15] Hobbs, D.S., and Dorschner, T.A., "Fourier Lithography: A New Manufacturing Tool for Optics", Workshop For Electro-Optics Manufacturing Science and Technology, Army Night Vision Labs, 95-498, May 1995

[16] Kaplan, S.G., Hanssen, L.M., and Datla, R.U., "Testing the Radiometric Accuracy of Fourier Transform Infrared Transmittance Measurements”, APPLIED OPTICS, Vol. 36, No. 34, pp 8896, December 1997 